\title{
A Case of Persistent Right Ventricular Failure after Rapid Decompression of a Large Chronic Pericardial Effusion
}

\author{
Harry Klimis, MBBS, BMedSci, Mikhail Altman, MD, FRACP, DDU, PhD, \\ Timothy Tan, MBBS, BSc, PhD, FRACP, FCSANZ, FACC, FESC, FAHA, \\ Jojie Natividad, BSc, MD, Robert Abraham, BSc, MBBS, FRACP, FCSANZ, \\ and Liza Thomas, MBBS, FRACP, PhD, Camperdown, Westmead, and Sydney, Australia
}

\section{INTRODUCTION}

We report the case of a patient with Hodgkin's lymphoma (diagnosis unknown at initial presentation) who presented with severe dyspnea secondary to a very large global pericardial effusion. Following pericardial drainage, the patient developed overt right ventricular (RV) failure. A detailed case report and brief overview of the potential mechanisms of persistent RV failure following pericardiocentesis are reported.

\section{CASE PRESENTATION}

A 33-year-old man presented to the emergency department with right-sided pleuritic chest pain, exertional dyspnea, and lower limb edema over the preceding 2 months. There was no significant medical history, and he was not on any regular medications. He was tachycardic (heart rate 130 beats/min) but normotensive (blood pressure $123 / 83 \mathrm{~mm} \mathrm{Hg}$ ) and tachypneic (30 breaths/min) with normal oxygen saturation on room air. His heart sounds were muffled, and neck veins were distended, with an elevated jugular venous pressure. Pulsus paradoxus was not assessed. Electrocardiography showed sinus tachycardia with low-voltage QRS and electrical alternans. Chest radiography showed marked cardiomegaly and a possible mass along the right mid to upper lateral cardiac border (Figure 1). Urgent transthoracic echocardiography (TTE) demonstrated a very large global pericardial effusion measuring at most $50 \mathrm{~mm}$ (during diastole), with early diastolic RV collapse indicating early echocardiographic evidence of tamponade (Figure 2, Video 1). RV and left ventricular (LV) systolic function was normal. The inferior vena cava was dilated, measuring $4.5 \mathrm{~cm}$, with $<50 \%$ collapse on inspiration. During urgent pericardiocentesis, $2 \mathrm{~L}$ of straw-colored fluid was removed via the subxiphoid approach. A pigtail catheter was left in situ on free drainage.

From the University of Sydney (H.K., M.A., L.T.); The George Institute for Global Health (H.K.), Camperdown; the Department of Cardiology Westmead Hospital, Westmead (H.K., M.A., T.T., J.N., R.A.); and the University of New South Wales (L.T.), Sydney, Australia.

Keywords: Cardiac tamponade, Pericardial decompression syndrome, Pericardiocentesis, Right ventricular failure, Pericardial effusion

Conflicts of interest: The authors reported no actual or potential conflicts of interest relative to this document.

Copyright 2018 by the American Society of Echocardiography. Published by Elsevier Inc. This is an open access article under the CC BY-NC-ND license (http://creativecommons.org/licenses/by-nc-nd/4.0/).

2468-6441

https://doi.org/10.1016/j.case.2018.02.001

142
Immediately after the procedure, heart rate was 118 beats/min and blood pressure was 133/83 mm Hg. Limited TTE performed immediately after drainage showed complete resolution of the pericardial effusion and a moderately dilated right ventricle with severely impaired systolic function (Figure 3). However, despite drainage of the pericardial fluid, the patient remained symptomatic with persistent dyspnea. Respiratory rate was 27 breaths/min, and oxygen saturation was $93 \%$ on room air, necessitating oxygen supplementation. Over the next few hours, the patient continued to be tachycardic, and blood pressure decreased to $90 / 60 \mathrm{~mm} \mathrm{Hg}$. Inotropic support was required to maintain normal blood pressure.

Progress TTE on day 1 after pericardiocentesis showed reaccumulation of a moderate $(22 \mathrm{~mm})$ residual pericardial effusion. There was no evidence of RV diastolic collapse as noted previously. However, the right ventricle was moderately dilated (basal diameter $54 \mathrm{~mm}$ ) with severely impaired segmental systolic dysfunction (Figure 4, Videos 2 and 3). The RV basal segment was akinetic with hyperkinetic mid-apical RV free wall segments, appearance typical for McConnell's sign. The interventricular septum was flattened and showed paradoxical motion. LV size was normal with mildly impaired systolic function. Additionally, there was no evidence of intracardiac shunt, and tricuspid regurgitant jet was insufficient to estimate pulmonary pressures. The inferior vena cava was not dilated and collapsed $>50 \%$ during inspiration, and there was trivial tricuspid regurgitation.

Computed tomographic pulmonary angiography (Figure 5) showed a large anterior mediastinal mass $(10 \times 18 \times 14 \mathrm{~cm})$ displacing the heart to the left and vessels posteriorly with associated large pericardial and a small right pleural effusion. The superior vena cava, right-sided pulmonary veins, and left atrium were partially compressed. However, the diameters of the main, right, and left pulmonary arteries were normal. Lymphoproliferative disease was suspected and remained high on the differential list. There was no evidence of a pulmonary embolus or features of pulmonary arterial hypertension. It was decided to proceed with surgical creation of a pericardial window because of evidence of reaccumulation of the pericardial effusion. Because of hypotension from severe RV systolic dysfunction coupled with anesthetic requirement, adrenaline and milrinone infusions were required during the procedure. The pericardial fluid had a yellow, turbid macroscopic appearance, and the analysis revealed a mixed inflammatory exudate comprising lymphocytes, neutrophils, and occasional eosinophils; no malignant cells were detected. Macroscopic appearance of the pericardium was normal, and microscopic appearance showed minor reactive changes only.

The patient proceeded to undergo computed tomography (CT)guided biopsy of the mediastinal mass 2 days later through a rightsided anterior thoracotomy, which revealed Hodgkin's lymphoma 


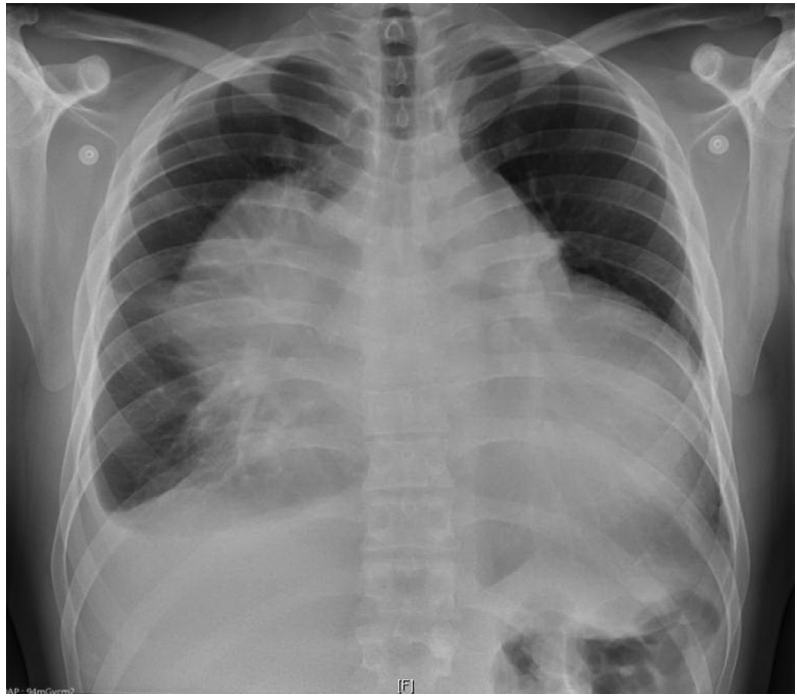

Figure 1 Plain posterior-anterior chest $x$-ray obtained in the emergency department on the day of presentation. There is marked cardiomegaly with bulging of the lateral right mid to upper cardiac margin. A small pleural effusion is present at the right lung base. No features of pulmonary edema or focal consolidations are seen.

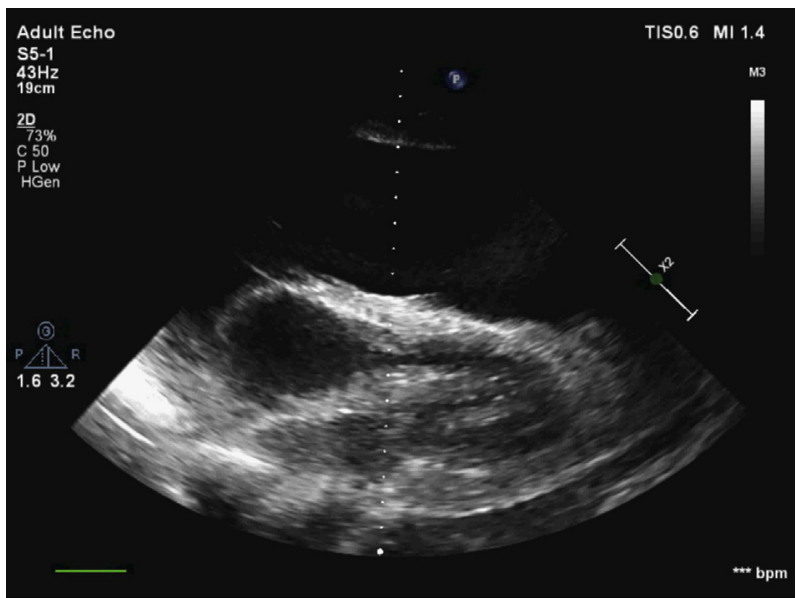

Figure 2 Urgent TTE performed on presentation (subcostal view). A very large global pericardial effusion measuring at most $50 \mathrm{~mm}$ (during diastole) is seen with early diastolic right ventricular collapse and echocardiographic evidence of tamponade.

(classical nodular sclerosing type). Staging positron emission tomography and CT of the abdomen and pelvis showed stage Ila disease (a single lymph node involved in addition to the mediastinal mass and no involvement below the diaphragm), and treatment with standard chemotherapy and radiotherapy was initiated. The patient was discharged 18 days following his initial presentation. Follow-up TTE 12 months after discharge showed normal biventricular size and function.

\section{DISCUSSION}

This case highlights the occurrence of pericardial decompression syndrome (PDS) with rapid drainage of a large pericardial effusion.

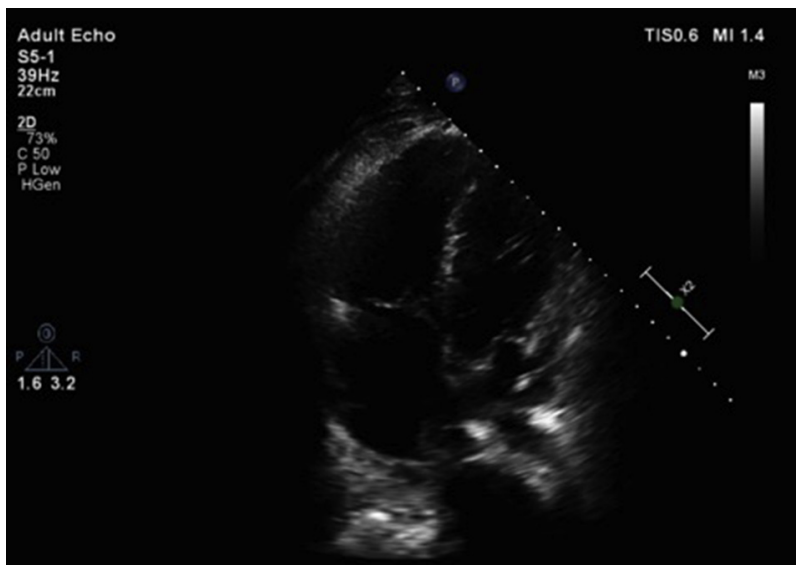

Figure 3 TTE performed immediately after pericardiocentesis (apical four-chamber view). Complete resolution of pericardial effusion was noted immediately after pericardiocentesis; the $\mathrm{RV}$ was moderately dilated with severe systolic impairment.

Although in the vast majority of cases, drainage of pericardial effusion occurs without any significant compromise of ventricular function, PDS can occur in some instances and therefore necessitates recognition of its occurrence. In our case, the cause of the effusion was likely nonmalignant, and given the presence of an inflammatory milieu on pericardial fluid analysis and absence of opportunistic infection, the cause of the pericardial effusion is likely to be inflammatory or reactive. Partial superior vena cava obstruction, as was seen in this case, could also potentially explain the pericardial and pleural effusions. However, in this instance a transudate would have been expected on fluid analysis. A paraneoplastic process is also a possible differential diagnosis.

Pericardiocentesis is a lifesaving procedure in the situation of cardiac tamponade, aimed at rapidly reducing the pericardial pressures. However, complication rates as high as $10 \%$ have been reported and include cardiac puncture, coronary artery injuries, arrhythmias, pneumothorax, hemothorax, pneumopericardium, and hepatic injury. ${ }^{1}$ Interestingly, there have been several, albeit infrequent, reports of paradoxical hemodynamic compromise manifesting as $\mathrm{RV}^{2-4} \mathrm{LV}^{5,6}$ or biventricular ${ }^{7-10}$ failure following pericardial drainage. Cases of normal biventricular function and acute pulmonary edema have also been reported. ${ }^{11-13}$ PDS is defined as hemodynamic compromise and/or pulmonary edema paradoxically occurring soon after successful pericardial fluid drainage, which is often associated with ventricular impairment. ${ }^{13,14}$ Treatment is supportive, and ventricular function is expected to recover in survivors.

A recent review of case reports found a total of 35 cases of PDS ${ }^{14}$ with onset varying from immediately after drainage to 48 hours later. The causes of the effusion were unknown in the majority of cases ( $n=15[42 \%]$ ), with the second most common being malignant effusion ( $n=14$ [40\%]). The volume of pericardial fluid drained ranged from 450 to $2,100 \mathrm{~mL}$ (mean, $888 \mathrm{~mL}$ ). Presentations of PDS were predominantly LV failure $(n=14$ [40\%]) and cardiogenic pulmonary edema without shock $(n=10$ [29\%]). RV and biventricular failures were less frequent presentations. There was a high mortality rate in this case series $(n=10$ [29\%]), which occurred between 6 hours and 14 days after drainage. Interestingly, mortality was associated only with surgical 


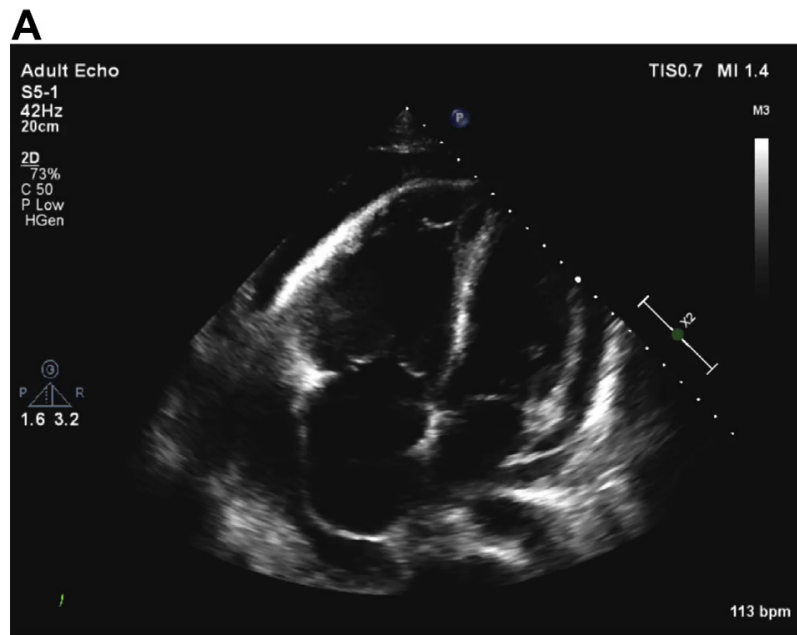

\section{B}

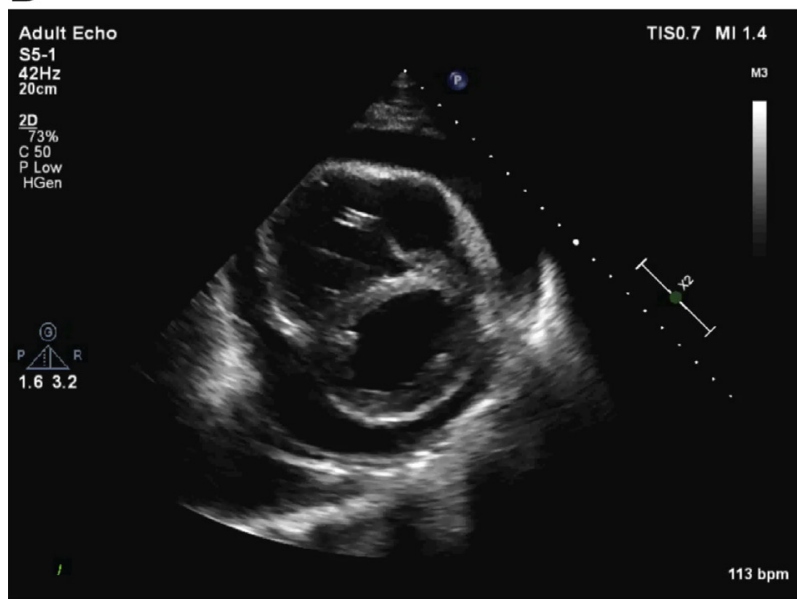

Figure 4 Progress TTE on day 1. Apical four-chamber (A) and parasternal short-axis (B) views. Images taken in end-diastole. The images show a moderate $(22 \mathrm{~mm})$ residual pericardial effusion with no evidence of tamponade, but the RV was moderately dilated (base $54 \mathrm{~mm}$ ) with severely impaired segmental right ventricular systolic dysfunction. There is evidence of septal flattening in diastole consistent with right ventricular volume overload (B).

drainage, with no deaths in those who underwent pericardiocentesis. In those with LV impairment who survived and had follow-up TTE, LV function normalized. Similarly, reports of predominant $\mathrm{RV}$ dysfunction also demonstrate recovery of RV systolic function in those who survived following development of PDS. ${ }^{2-4}$

In our case, McConnell's sign was present, which has been described as hypo- or akinesis of the basal to mid RV free wall with preservation of RV apical function. This sign is usually associated with an acute pulmonary embolus, though not synonymous, and if present in the absence of pulmonary embolus may suggest pulmonary hypertension. ${ }^{15}$ In our case, pulmonary pressures were not elevated. There have been no previous reports of McConnell's sign being present in PDS. As such, the mechanism of McConnell's sign is not well understood. In our case, we hypothesized that localized ischemia of the RV free wall, as a result of increased wall stress following rapid decompression of the pericardial effusion, and the resultant volume load on the right ventricle, could have contributed to its development. ${ }^{16}$

The mechanism of PDS is unclear, without any single pathologic pathway being elucidated; however, there are many proposed mechanisms. ${ }^{17}$ Early descriptions have postulated that rapid drainage of a large pericardial effusion would decompress the right-sided chambers and in turn cause a marked increase in venous return. This would result in overloading of the ventricles, culminating in reduced ventricular contractility due to overstretched myocardial sarcomeres, while systemic vascular resistance remained elevated despite treatment of cardiac tamponade. 3,13 Similarly, Anguera et $\mathrm{al}^{2}$ postulated that rapid venous return following pericardial drainage may cause rapid dilatation of the right ventricle, resulting in myocardial damage. Others have suggested a reduction in coronary perfusion pressures (due to elevated pericardial pressures that persist following rapid decompression) may lead to myocardial stunning and hibernation with resultant transient ventricular systolic dysfunction. ${ }^{2,5,9,10,14}$ In our case, given the rapid large volume pericardiocentesis that occurred, the presence of McConnell's sign and subsequent recovery of ventricular function suggest that acute myocardial stunning from reduction in coronary perfusion pressures may be the underlying mechanism of PDS. This manifested primarily as acute RV systolic dysfunction, resulting in hypotension requiring inotropic support.

Another hypothesized mechanism is the unmasking of occult systolic impairment, such as from malignant myocardial infiltration, when treating cardiac tamponade. Unmasking of latent dysfunction may occur because of removal of the sympathetic drive, which produced transient tachycardia and inotropic effect. ${ }^{10}$ However, this is unlikely to be the case when systolic impairment improves rapidly with supportive treatment.

In our case, there was partial obstruction of the left atrium and right-sided pulmonary veins, which may also explain RV dilatation and systolic impairment. However, the main pulmonary artery when measured on CT was normal in size, indicating that it was not transmitted pressure from partial compression of the pulmonary veins that resulted in RV dysfunction. Unfortunately, no follow-up CT was performed at 12 months to determine if the improved RV function seen on follow-up TTE correlated with reduction in the size of the mass and degree of left atrial compression.

Because of its rarity, occurrence with varying amounts of pericardial collections, and development even with drainage of relatively small volumes of pericardial fluid, there are no guidelines that determine a "safe" amount of fluid that can be drained to avoid the development of PDS. It has been proposed that pericardial effusions be drained partially until the disappearance of signs of tamponade, with subsequent more gradual and prolonged drainage of pericardial fluid. ${ }^{17}$ However, there is a lack of substantive data to support this.

\section{CONCLUSIONS}

PDS is rare and has a high mortality rate. The true incidence of PDS is unknown because of underrecognition of this condition. Its nonuniform clinical presentation and confounding from other causes of hemodynamic compromise may delay diagnosis. Survivors are expected to have spontaneous recovery of ventricular function following supportive therapy. 

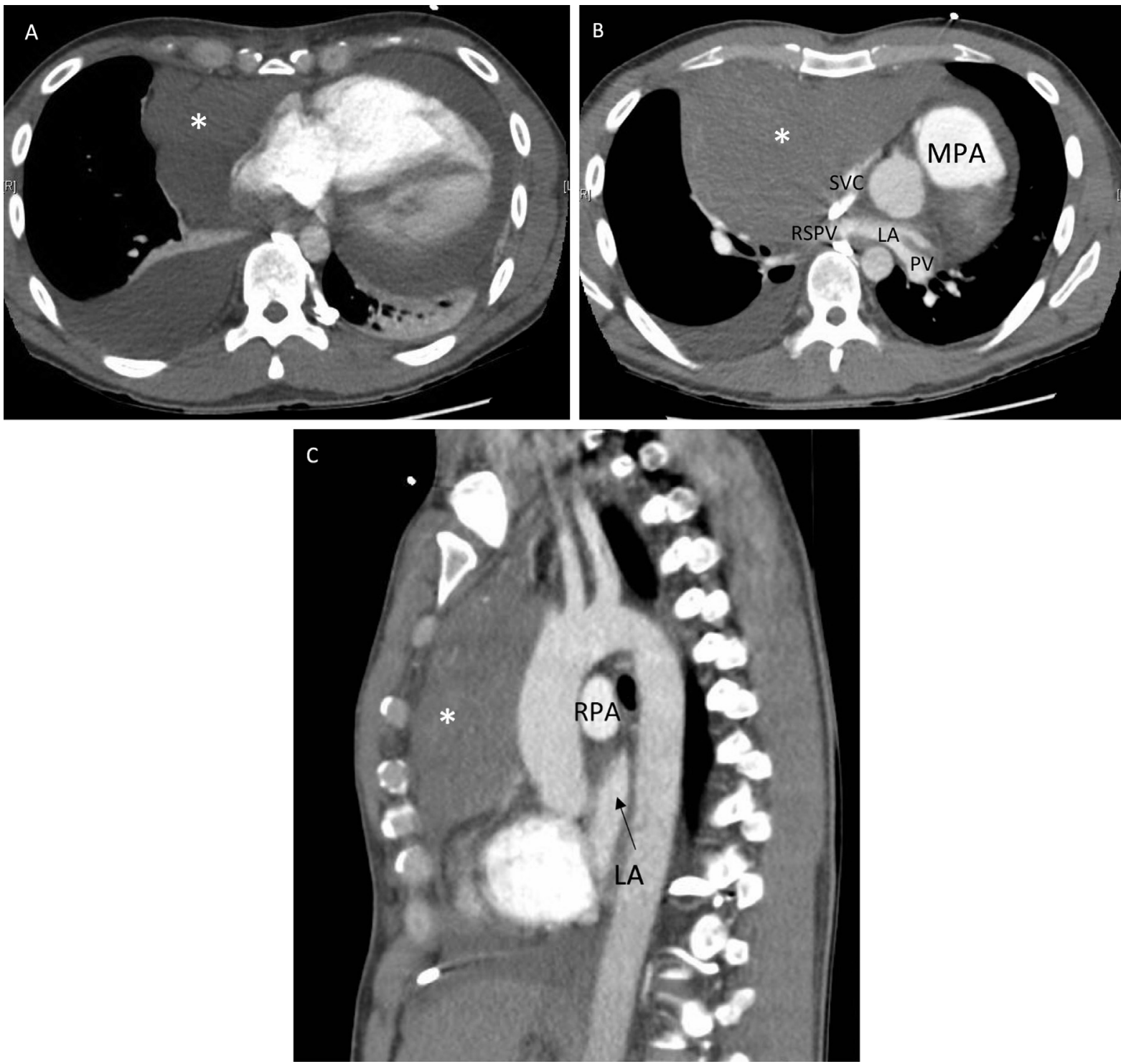

Figure 5 Computed tomographic pulmonary angiogram. Panels A and $\mathbf{B}$ depict axial planes at the level of the ventricles and main pulmonary artery, respectively. Panel $\mathbf{C}$ depicts the sagittal plane. Visible is a large anterior mediastinal mass measuring $10 \times 18$ $\times 14 \mathrm{~cm}$ (asterisk) displacing the heart to the left and vessels posteriorly with associated large pericardial and right small pleural effusion (A). The main pulmonary trunk (MPA, B) measures $2.7 \mathrm{~cm}$ in diameter, whereas the intrapericardial segments of the right pulmonary artery (RPA; C) and left pulmonary artery measure $1.4 \mathrm{~cm}$ in diameter, with no features of pulmonary arterial hypertension apart from some dilation of the right cardiac chambers $(\mathbf{A})$. The left atrium (LA), right-sided pulmonary veins (RSPV), and superior vena cava (SVC) are partially compressed (B). PV, Pulmonary veins; RPA, right pulmonary artery.

\section{SUPPLEMENTARY DATA}

Supplementary data related to this article can be found at https://doi. org/10.1016/j.case.2018.02.001.

\section{REFERENCES}

1. Adler Y, Charron P, Imazio M, Badano L, Barón-Esquivias G, Bogaert J, et al. 2015 ESC guidelines for the diagnosis and management of pericardial diseases: the task force for the diagnosis and management of pericardial diseases of the European Society of Cardiology (ESC) endorsed by: the European Association for Cardio-Thoracic Surgery (EACTS). Eur Heart J 2015:36:2921-64.
2. Anguera I, Pare C, Perez-Villa F. Severe right ventricular dysfunction following pericardiocentesis for cardiac tamponade. Int J Cardiol 1997 59:212-4

3. Kuroda M, Amano M, Enomoto S, Miyake M, Kondo H, Tamura T, et al Severe right ventricular and tricuspid valve dysfunction after pericardiocentesis. J Med Ultrason (2001) 2016;43:533-6.

4. Liao BT, Lo SS. Paradoxical hemodynamic collapse after subxiphoid peri cardial window. A A Case Rep 2015;4:65-7.

5. Sevimli S, Arslan S, Gundogdu F, Senocak H. Development of left ventric ular apical akinesis and thrombus during pericardiocentesis for pericardial tamponade. Turk Kardiyol Dern Ars 2008;36:338-41.

6. Versaci F, Donati R, Mezzanotte R, Chiariello L, Ammirati F. An unusual complication following pericardiocentesis: reversible left ventricular dysfunction. J Cardiovasc Med (Hagerstown) 2015;16(suppl 2):S133-5. 
7. Al Banna R, Husain A. Reversible severe biventricular dysfunction postpericardiocentesis for tuberculous pericardial tamponade. BMJ Case Rep 2011. pii: bcr0220113837.

8. Koerner MM, Alam M, El-Banayosy A, Misra A, Wall MJ Jr, Zeriouh M, et al. A case of biventricular failure after pericardial window for large pericardial effusion. Heart Surg Forum 2015;18:E36-7.

9. Ligero C, Leta R, Bayes-Genis A. Transient biventricular dysfunction following pericardiocentesis. Eur J Heart Fail 2006;8:102-4.

10. Wolfe MW, Edelman ER. Transient systolic dysfunction after relief of cardiac tamponade. Ann Intern Med 1993;119:42-4.

11. Abdelsalam M, Moritz TA, Snyder JA, Cheriyath P, Spizzieri CL. Paradoxical hemodynamic instability complicating pericardial window surgery for cardiac tamponade in a cancer patient. Tex Heart Inst J 2012;39:711-3.

12. Fozing T, Zouri N, Adam O, Oezbek C. Management of a patient with pericardial decompression syndrome and HOCM. BMJ Case Rep 2016. pii: bcr2015211550.
13. Vandyke WH Jr, Cure J, Chakko CS, Gheorghiade M. Pulmonary edema after pericardiocentesis for cardiac tamponade. N Engl J Med 1983;309: 595-6.

14. Pradhan R, Okabe T, Yoshida K, Angouras DC, DeCaro MV, Marhefka GD. Patient characteristics and predictors of mortality associated with pericardial decompression syndrome: a comprehensive analysis of published cases. Eur Heart J Acute Cardiovasc Care 2015;4:113-20.

15. Mediratta A, Addetia K, Medvedofsky D, Gomberg-Maitland M, Mor-Avi V, Lang RM. Echocardiographic diagnosis of acute pulmonary embolism in patients with McConnell's sign. Echocardiography 2016;33:696-702.

16. McConnell MV, Solomon SD, Rayan ME, Come PC, Goldhaber SZ, Lee RT. Regional right ventricular dysfunction detected by echocardiography in acute pulmonary embolism. Am J Cardiol 1996;78:469-73.

17. Imazio M. Pericardial decompression syndrome: a rare but potentially fatal complication of pericardial drainage to be recognized and prevented. Eur Heart J Acute Cardiovasc Care 2015;4:121-3. 